\title{
Using ARAMIS system for measurement of structural stability of running wind tunnel
}

\author{
Viatlii Yanovych ${ }^{1,1}$, Daniel Duda ${ }^{1}$ \\ ${ }^{1}$ Faculty of Mechanical Engineering, University of West Bohemia, Department of Power System \\ Engineering, Univerzitní 2732/8, 30100 Pilsen, Czech Republic
}

\begin{abstract}
Wind tunnels are popularly used to study various aerodynamic phenomena. Their design significantly influences the quality of the created airflow. During the flow generation, the construction parts of the wind tunnel to get deformed due to pressure variations. This phenomenon significantly complicates the creation of high-level laminar flow and worsens the results of experimental studies. The main purpose of this work is to investigate the effect of a sharp change in flow velocity on the structural stability of parts of a wind tunnel with a capacity of $55 \mathrm{~kW}$. For experimental studies, we used the ARAMIS optical measurement system. Using it, we estimated the amount of deformation and mutual displacement of parts of the running wind tunnel at a flow rate of $76 \mathrm{~m} \cdot \mathrm{s}^{-1}$.
\end{abstract}

\section{Introduction}

The development of low-speed experimental aerodynamics continues to evolve. This is very important for the development of different machines and other devices that operate under the influence of airflow. In 1960-1980, researchers have long believed that the place of subsonic wind tunnel will take computer simulation [1]. Of course, computational simulations have improved since then, but they have not come close to reaching the level to replace real experimental studies. Therefore, in most cases, modern researchers use comprehensive methods of analyzing the phenomena of Fluid Mechanics. An integral part of the real experiment is high-quality technological equipment. The main part of which is a wind tunnel with a high degree of laminar flow. Many scientific institutions are developing their wind tunnels.

For example in Article [2] describes the development of a multi-purpose wind tunnel with a capacity of $15 \mathrm{~kW}$. This wind tunnel was built at the Faculty of Engineering and Built Environment, Universiti Kebangsaan, (Malaysia). Computational Fluid Dynamics simulation was used to establish the optimal geometric parameters of the tunnel and the characteristics of its flow. However, a description of the reporting measuring equipment was used to estimate the actual parameters of the generated flow.

Article [3] presents the results of a study of a new wind tunnel developed by the University of Applied Sciences in Saarbrücken (Germany). This tunnel has a closed type of Göttinger construction. The air is driven by a rotor with a capacity of $75 \mathrm{~kW}$, located on the

${ }^{1}$ Corresponding author: yanovych@,kke.zcu.cz 
upper side. The Finite Element Analysis method was used to study the characteristics of tunnel construction. This method helped to found that in different parts of the tunnel construction there are different values deformation.

There are also a large number of low-speed open-type wind tunnels [4-7]. That is, these tunnels do not have a closed-loop for airflow. This type of construction is common. In the article [8] shown a small subsonic wind tunnel with an open circuit. The maximum flow velocity in the test section is $20 \mathrm{~m} \cdot \mathrm{s}^{-1}$, the compression ratio is 6.25 and creates maximum uniformity in the middle plane of the working section. Among the main parts of this design is a section for generating and calming the air.

One of the most common practical tasks that are solved with the help of a wind tunnel is to study the patterns of behavior of airflow around various objects. In particular, many researchers have focused on the study of turbulence [9-13]. However, it should be noted that only wind tunnels that have a high quality of the generated flow can provide adequate experimental results.

Nowadays, systems for non-contact measurement of material deformation have become widespread. These systems are very practical. Because they are making it possible to analyze the deformation of objects with complex geometry under the action of airflow.

For example, in article [14] shown the results of experimental studies of the deformation of the wings of two models of supersonic aircraft. During tests in a 9-by-7-foot supersonic wind tunnel at NASA's Ames Research Center. The method of stereophotogrammetry was used for experimental research. Measurements of deformations of surfaces of test models were carried out due to the simultaneous shooting of high-speed cameras from two directions. The obtained results allowed to estimate the bending and twisting parts of the studied models at different flow velocity.

A similar method was used by the authors in Article [15]. Where they developed a videogrammetry method for measuring deformation, which is based on the use of paint that has a high sensitivity to pressure. They propose to use this technique to estimate unstable air-elastic deformation and pressure fields on the wings of an airplane in transcranial and supersonic wind tunnels.

The main task of the wind tunnel is to generate a low degree of turbulence of the output airflow. It is clear that this parameter significantly depends on the design quality of the wind tunnel. The design of the tunnel must withstand sharp pressure drops and impulses of airflow. Vibration can also be attributed to the negative phenomena of any mechanical system. This phenomenon can occur if the rotating parts of the tunnel structure (such as an electric motor or a radial fan) have some eccentricity. In this case, the vibration will be transmitted to the entire body of the wind tunnel and will significantly degrade the quality of airflow. That is why the study of deformation and characteristic of mutual displacement of the main parts of the wind tunnel construction is an important task.

\section{Materials and methods of research}

An open-type wind tunnel was developed in the laboratory of power machines of the University of Western Bohemia (Fig. 1 a) $[16,17]$. This wind tunnel can generate an flow velocity of up to $U=90 \mathrm{~m} \cdot \mathrm{s}^{-1}$.

Among the main parts of the structure are the section for generating airflow, diffuser, settling chamber, confuser (contraction), and the test section. All parts of the wind tunnel except the flow generation section are located on one common frame.

The flow generation section has a three-phase Tamel electric motor with a capacity of $55 \mathrm{~kW}$. A radial fan is attached to the motor shaft. The air enters the wind tunnel through a cylindrical filter. Then the airflow is going to the diffuser. After passing the last, the value velocity of a flow decreases but the static pressure increases. The diffuser is divided into 
three sections. Between these parts are metal screens for calm the airflow. A flexible connection was used to prevent the transmission of vibration from the flow generation section to the diffuser.

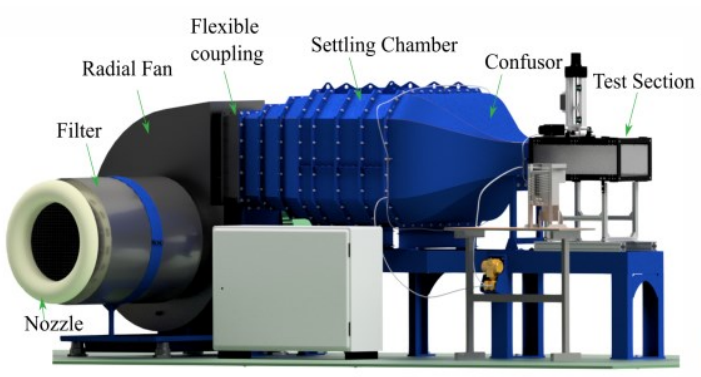

a)

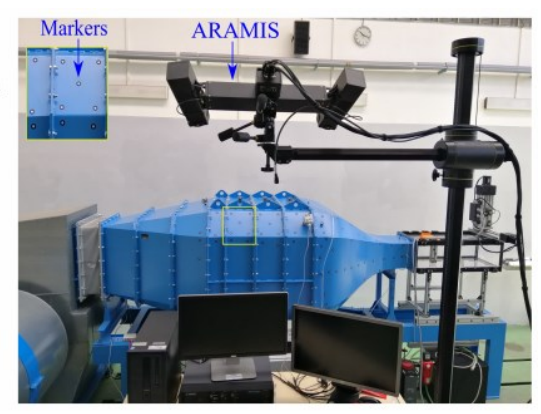

b)

Fig. 1. Construction of the wind tunnel and optical measuring system ARAMIS. a) General view of the wind tunnel. b) Analysis of structural deformation of the wind tunnel.

The settling chamber has a cross-section of $740 \mathrm{~mm}$. The purpose of this module is to eliminate the turbulence of the flow, due to the destruction of air vortices that cause the pulse of the flow velocity. For this, the settling chamber space is divided into four separate sections, between which are installed metal screens. Each section is a steel body and allows you to equalize the pressure at the top and bottom part of the chamber. To avoid unwinding of the flow along its axis in the first section of the chamber is honeycomb in the form of a hexagonal membrane.

After the settling chamber the confuser is placed. It should be noted that at the outlet of the confuser the flow velocity increases significantly but decreases the static pressure. From a structural point of view, it is a metal structure with a complex configuration that ends with a suitable outlet for the test section. The confuser output is connected to the testing section. The parameters of the wind tunnel were controlled using the developed program in the LabVIEW.

We used pressure indicator Druck DPI 800 for identification the pressure drop in the confuser.

We used the non-contact optical measuring system ARAMIS to measure the structural deformation and amount of mutual displacement of parts of the wind tunnel (Fig. $1 \mathrm{~b}$ ). This system has two stereo cameras that provide active accurate monitoring of objects in threedimensional coordinates on the principle of triangulation. It performs high-precision 3D measurement in the submicrometer range, regardless of the geometry and temperature of the sample. In our case, the ARAMIS SRX system from the ARAMIS sensor family was used.

This system consists of two 12-megapixel cameras that have a maximum image recording frequency of $335 \mathrm{~Hz}$. Also, by reducing the image quality, we have increased the recording frequency to $500 \mathrm{~Hz}$. Based on the received data from the image surface components or reference points are formed. These points are analyzed for threedimensional displacements depending on time. In our case, markers with a diameter of 18 $\mathrm{mm}$ were used to identify reference points. The cameras were placed opposite each other on a $600 \mathrm{~mm}$ long frame.

To analyze the behavior of the wind tunnel construction, three parts were selected: the the confuser, the settling chamber and flexible coupling.

Experimental studies were performed for two cases. In the first case, the behavior of the object was studying at a sharp increase $U$ from 0 to $76 \mathrm{~m} \cdot \mathrm{s}^{-1}$ and its decrease from $76 \mathrm{~m} \cdot \mathrm{s}^{-1}$ 
Recording frequency was $\lambda=3-5 \mathrm{~Hz}$. In the second case flow velocity was constant $U=76$ $\mathrm{m} \cdot \mathrm{s}^{-1}$ and recording frequency was $\lambda=500 \mathrm{~Hz}$.

\section{Results and discussion}

The ARAMIS system allows us, among others, to analyze the change in distance between two reference points. It is this distance that we will estimate in further analysis.

The deformation of the confuser construction depending on the flow velocity is shown in Fig. 2. For this analysis, two measuring lines $L 1$ and $L 2$ were generated between the four reference points of the confuser. These lines, respectively, characterize the deformation of the confuser relative to its height and length. Experimental studies were performed with a sharp increase of $U$ to $76 \mathrm{~m} \cdot \mathrm{s}^{-1}$ and a subsequent sharp decrease of $U$ to $5 \mathrm{~m} \cdot \mathrm{s}^{-1}$. The recording frequency of the cameras was $\lambda=3 \mathrm{~Hz}$. As we can see, the maximum values of the deformation of the confuser relative to its height and length are observed at different flow velocity (Fig. 2 b). We can see that, when the value of $\delta_{1}$ increases, the value of $\delta_{2}$ decreases. The maximum deformation for $\delta_{1} \approx 0.035 \mathrm{~mm}$ at $40 \mathrm{~m} \cdot \mathrm{s}^{-1}$. Whereas the maximum value of $\delta_{1} \approx 0.03 \mathrm{~mm}$ at $U=76 \mathrm{~m} \cdot \mathrm{s}^{-1}$. It is clear that the difference between the values obtained is very small.

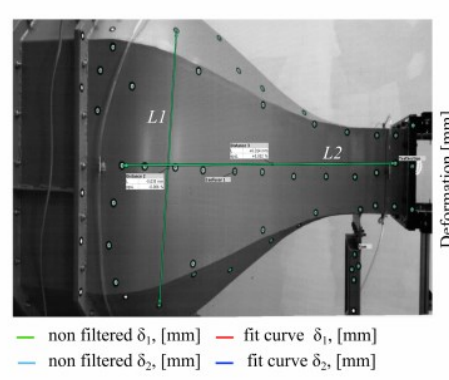

a)

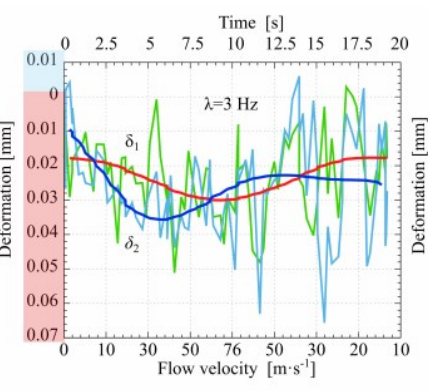

b)

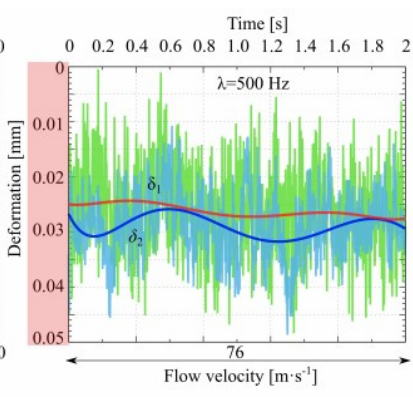

c)

Fig. 2. Deformation of the confuser construction depending on the flow velocity. a) Measuring lines between the reference points of the confuser. $L 4$ and $L 3$ is responsible for longitudinal and height deformation of the confuser. b) The magnitude of the deformation at a sharp increase $U$ from 0 to 76 $\mathrm{m} \cdot \mathrm{s}^{-1}$ and its decrease from $76 \mathrm{~m} \cdot \mathrm{s}^{-1}$ to $5 \mathrm{~m} \cdot \mathrm{s}^{-1}$. c) The magnitude of deformation at a constant flow velocity $76 \mathrm{~m} \cdot \mathrm{s}^{-1}$. The blue area shows the elongation of the measuring line, red area of its reduction.

But the obtained distribution is quite logical and have a physical meaning. From Fig. $2 \mathrm{~b}$ we can see that with increasing flow velocity from the beginning there is a significant increase in deformation in the longitudinal direction. This is understandable because the static pressure at the beginning of the confuser is much higher than at the outlet. The difference between these pressures is approximately $2.4 \mathrm{kPa}$. Therefore, at first there is a slight elongation of the confuser. After that, take into account the regularity of uniform distribution of stresses in the material, the process of deformation of the confuser relative to its height begins. A further increase in the value of $\delta_{1}$ will lead to a decrease in the value of $\delta_{2}$. That is, there is a process of mutual compensation of the amount of deformation of the confuser in accordance with its length and height. At a constant flow rate, the amount of deformation of the confuser is equal to of $\delta_{1} \approx 0.025 \mathrm{~mm}$ at $U=76 \mathrm{~m}^{-1} \mathrm{~s}^{-1}$ and $\delta_{2} \approx 0.03 \mathrm{~mm}$ at $U=76 \mathrm{~m} \cdot \mathrm{s}^{-1}$.

One of the main parts of the wind tunnel design is the settling chamber. The behavior of its construction under the action of pressure drops is an important factor in generating airflow with a low degree of turbulence. In Fig. 3 shows the results of experimental studies of the deformation of the settling chamber depending on the different flow rate. 


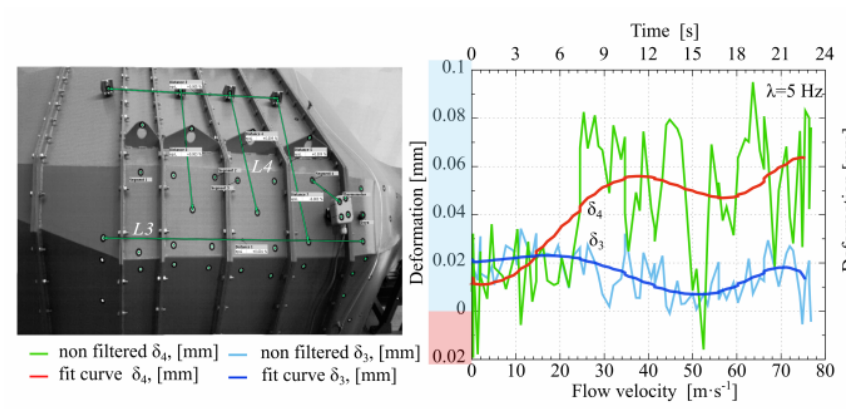

a)

b)

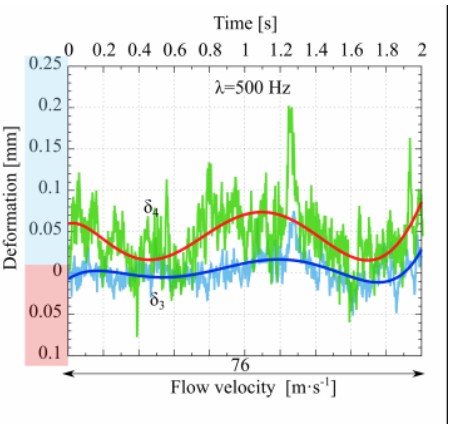

c)

Fig. 3. Observe of deformation of the settling chamber. a) Measuring lines between the reference points of the settling chamber. Measuring line $L 3$ is responsible for longitudinal deformation. Measuring line $L 4$ is responsible for the deformation relative to the width of the settling chamber. b) The magnitude of deformation at a sharp increase $U$ from 0 to $76 \mathrm{~m} \cdot \mathrm{s}^{-1}$. c) The magnitude of deformation at $U=76 \mathrm{~m} \cdot \mathrm{s}^{-1}$.

The experiments were performed with different camera recording frequencies. In the case of a sharp increase in flow velocity $\lambda=5 \mathrm{~Hz}$ (Fig. $3 \mathrm{~b}$ ), at a constant flow velocity $\lambda=500 \mathrm{~Hz}$ (Fig. $3 \mathrm{c}$ ). For experimental studies, two lines between reference points were generated. Accordingly, the first line $L 3$ characterizes the longitudinal deformation of the settling chamber $\delta_{3}$, the second line $L 4$ characterizes the deformation relative to its width $\delta_{4}$. The maximum value of deformation at $76 \mathrm{~m} \cdot \mathrm{s}^{-1}$ for $\delta_{3} \approx 0.02 \mathrm{~mm}$, for $\delta_{4} \approx 0.05 \mathrm{~mm}$. At a constant flow velocity of $76 \mathrm{~m} \cdot \mathrm{s}^{-1}$, the longitudinal deformation of $\delta_{3}$ actually disappears. The deformation $\delta_{4}$ has a sinusoidal distribution in the range of $\approx 0.02-0.07 \mathrm{~mm}$. We should be noted that the construction of the settling chamber has minimal deformation at a sharp increase in flow velocity. That allows us to provide qualitative calming of airflow.

The wind tunnel can be divided into two parts: part of the tunnel to generate airflow and part of the tunnel to calm it. A flexible coupling is used to connect them. It is clear that during the operation of the wind tunnel, the pressure difference in these parts can lead to some displacement. We investigated the displacement of the part of the tunnel to generate the flow relative to the part of the tunnel to calm the flow (Fig. 4).

We first generated five measuring lines between reference points. Three lines $L 5, L 6, L 7$ show the horizontal mutual displacement $\Delta L_{5}, \Delta L_{6}, \Delta L_{7}$ of the two parts of the tunnel construction. Two measuring lines $L 8$ and $L 9$ are placed symmetrically on the diagonals of the flexible coupling and show displacement $\Delta L_{8}, \Delta L_{9}$. For each measuring line, we were investigated two cases. In the first case, at a sharp at a sharp increase $U$ from 0 to $76 \mathrm{~m} \cdot \mathrm{s}^{-1}$ and its decrease from $76 \mathrm{~m} \cdot \mathrm{s}^{-1}$ to $5 \mathrm{~m} \cdot \mathrm{s}^{-1}$ at $\lambda=4 \mathrm{~Hz}$ (Fig. $4 \mathrm{~b}$, e). In the second case at a constant flow velocity at $\lambda=500 \mathrm{~Hz}$ (Fig. $4 \mathrm{c}, \mathrm{f}$ ).

Fig. $4 \mathrm{~b}$ shows that the maximum displacement with a sharp increase in flow velocity in the horizontal plane is observed at $76 \mathrm{~m} \cdot \mathrm{s}^{-1}$ for $\Delta L_{5} \approx 0.35 \mathrm{~mm}$. At a constant flow velocity (Fig. 4 e) the amount of displacement remains constant $\Delta L_{5} \approx 0.21 \mathrm{~mm}, \Delta L_{6} \approx 0.13 \mathrm{~mm}, \Delta L_{7}$ $\approx 0.1 \mathrm{~mm}$. Interestingly, the displacement values for $\Delta L_{6}$ and $\Delta L_{7}$ are different. Although they are placed symmetrically. This shows that the displacement occurs not only parallel to the horizontal plane. This fact is confirmed by experimental data for the displacement of $\Delta L_{8}$ and $\Delta L_{9}$ (Fig. 4 e). This shows that at a sharp increase in flow velocity, the obtained distribution of curves $\Delta L_{8}$ and $\Delta L_{9}$ is symmetric. Their maximum displacement is in the range of $0.6-0.7 \mathrm{~mm}$. At a constant flow velocity of $76 \mathrm{~m} \cdot \mathrm{s}^{-1}$, the displacement for $\Delta L_{8} \approx 0.6$ $\mathrm{mm}$ and for $\Delta L_{9} \approx 0.5 \mathrm{~mm}$ (Fig. $4 \mathrm{f}$ ). 


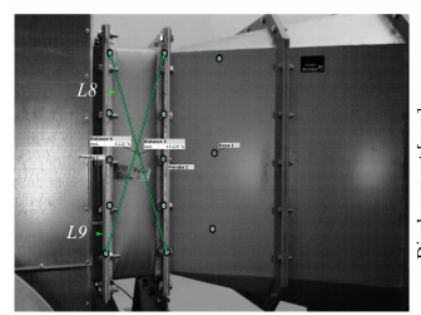

- fit curve $\Delta L_{8},[\mathrm{~mm}]-$ non filtered $\Delta L_{8},[\mathrm{~mm}]$ - fit curve $\Delta L_{9},[\mathrm{~mm}]$ - non filtered $\Delta L_{9},[\mathrm{~mm}]$

a)

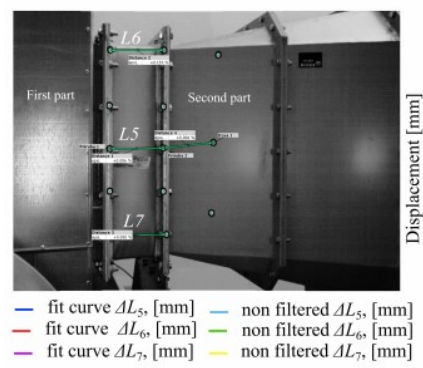

d)

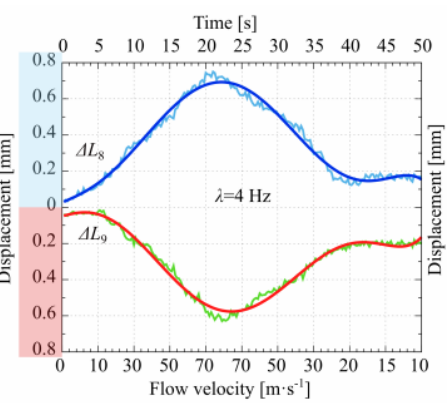

b)

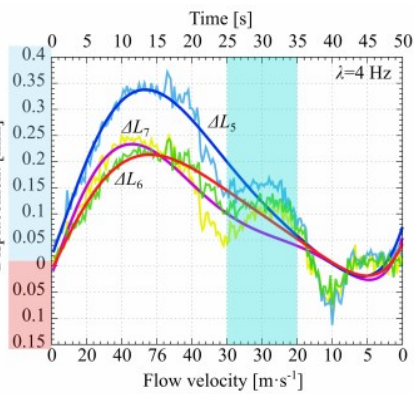

e)

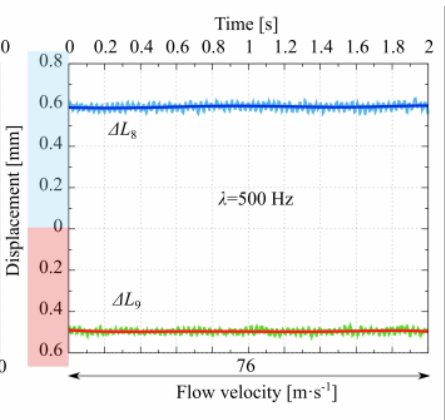

c)

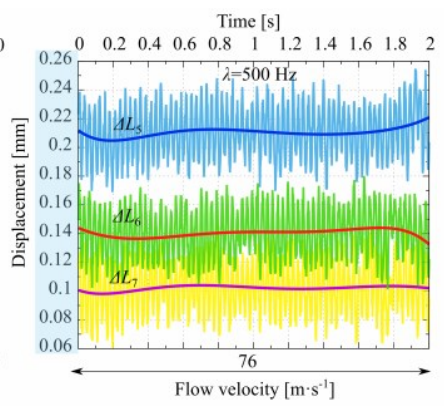

f)

Fig. 4. Estimation of relative mutual displacement of tunnel parts for generation and calming of airflow. a), d) Measuring lines between the reference points. $L 5, L 6, L 7$ is responsible for longitudinal displacement. $L 8$ and $L 9$ are responsible for the offset relative to the diagonals of the flexible coupling. b), e) The magnitude of displacement at a sharp increase $U$ from 0 to $76 \mathrm{~m} \cdot \mathrm{s}^{-1}$ and its decrease from $76 \mathrm{~m} \cdot \mathrm{s}^{-1}$ to $5 \mathrm{~m} \cdot \mathrm{s}^{-1}$. c), f) The magnitude of displacement at $U=76 \mathrm{~m} \cdot \mathrm{s}^{-1}$. The blue area shows the elongation of the measuring line, red area of its reduction.

In general, this phenomenon occurs due to a significant pressure drop at sharp putting air flow by a radial fan to part of the tunnel to calm the flow. The airflow receives some resistance when passing through the diffuser. Because after the diffuser in the settling chamber the pressure is much higher than atmospheric. Therefore, some parts of the flow to form the so-called reverse thrust. Which acts in the opposite direction and shifts the section to generate the flow relative to the vertical plane. The obtained results confirm the need to use a flexible coupling in the production of wind tunnels.

\section{Conclusion}

We used the ARAMIS system to study the behavior of the structural parts of the wind tunnel as a function of flow velocity. As a result of the experiment, the amount of displacement of the electrical motor, deformation of the confuser, of the settling chamber, and of the test section. We also investigated the mutual displacement of parts of the wind tunnel to generate and to calm the airflow.

Studies have been done for several cases. In the first case, we sharp increased the flow velocity to $76 \mathrm{~m} \cdot \mathrm{s}^{-1}$. In the second case, we investigated the behavior of the tunnel construction at $U=76 \mathrm{~m} \cdot \mathrm{s}^{-1}$. We also changed the recording frequency of the cameras $\lambda=3$ $5 \mathrm{~Hz}$ (for the first case) and $\lambda=500 \mathrm{~Hz}$ (for the second case).

As we can see the obtained experimental data for the two measurement cases are different. With a sharp increase in the flow rate, the values obtained are greater than with a 
constant flow rate. This is due to the relaxation properties of the material of the structural parts of the tunnel.

The largest value of displacement we can observe near the elastic coupling. In fact, this value shows how much the section for generating the flow is displaced from the section of the wind tunnel to calm the flow. This phenomenon occurs due to the pressure difference in the above sections.

In general, we can conclude that the construction of the developed wind tunnel has a very high stiffness to pressure drop.

Acknowledgement: The work was supported from ERDF under the project Research Cooperation for Higher Efficiency and Reliability of Blade Machines (LoStr) No. CZ.02.1.01/0.0/0.0/16_026/ 0008389.

\section{References}

1. W.H. Rae, A. Pope, Low-Speed Wind Tunnel Testing, (1984)

2. H. Zambri, A.W. Ghopa, A. Shahrir, G.M. Izhar, A.A. Amer, R. Mohd, Z. Rozli, M. Wan, M.M.R.A. Abu, A. Zulkhairi, W.M Hanna, Jurnal Teknologi, 78, 63 (2016)

3. F. Rückert, D. Lehser-Pfeffermann, T. Müller, F. Joos, D. Theis, (2018)

4. B. Celis, H.H. Ubbens, Procedia Engineering, 147, 98 (2016)

5. R.D. Mehta, P. Bradshaw, Aeronautical Journal, 83(827), 443 (1979)

6. P. Bradshaw, R. Pankhurst, Progress in Aerospace Sciences, 5(C), 1 (1964)

7. J.B. Barlow, W.H. Rae, A. Pope, Low-Speed Wind Tunnel Testing, 3rd edn, (1999)

8. A. Teseletso, M. Namoshe, N. Subaschandar, S. Kutua, International Biennial Conference, (2015)

9. V. Uruba, In Proceedings Topical Problems of Fluid Mechanics, (2015)

10. C. Tropea, A. Yarin, J.F. Foss, Springer Handbook of Experimental Fluid Mechanics, (Springer, Heidelberg, DE, 2007)

11. S.B. Pope, Turbulent flows (Cambridge University Press, New York, USA, 1982)

12. G.K. Batchelor, The Theory of Homogeneous Turbulence (Cambridge University Press, New York, USA, 1982)

13. M. Bourgoin, C. Baudet, S. Kharche et al., CEAS Aeronaut, 9(2), 269 (2018)

14. E.T. Schairer, L.K. Kushner, T.J. Garbeff, J.T. Heineck, AIAA Aerospace Sciences Meeting, Kissimmee, 1 (2015)

15. T. Liu, J. Montefort, J. Gregory, S. Palluconi, J. Crafton, S. Fonov, AIAA Fluid Dynamics Conference and Exhibit, 1 (2011)

16. V. Yanovych, D. Duda, V. Horacek, V. Uruba, AIP Conference Proceedings, 2189 (2019)

17. V. Yanovych, D. Duda, V. Horacek, V. Uruba, AIP Conference Proceedings, 2118 (2019) 